\title{
Normal-mode driven exploration of protein domain motions
}

\author{
Yves-Henri Sanejouand* \\ UFIP, UMR 6286 of CNRS, \\ Faculté des Sciences et des Techniques, Nantes, France.
}

March $22^{\text {th }}, 2021$

\begin{abstract}
Domain motions involved in the function of proteins can often be well described as a combination of motions along a handfull of low-frequency modes, that is, with the values of a few normal coordinates. This means that, when the functional motion of a protein is unknown, it should prove possible to predict it, since it amounts to guess a few values.

However, without the help of additional experimental data, using normal coordinates for generating accurate conformers far away from the initial one is not so straightforward. To do so, a new approach is proposed: instead of building conformers directly with the values of a subset of normal coordinates, they are built in two steps, the conformer built with normal coordinates being just used for defining a set of distance constraints, the final conformer being built so as to match them. Note that this approach amounts to transform the problem of generating accurate protein conformers using normal coordinates into a better known one: the distance-geometry problem, which is herein solved with the help of the ROSETTA software.

In the present study, this approach allowed to rebuild accurately six large amplitude conformational changes, using at most six low-frequency normal coordinates. As a consequence of the lowdimensionality of the corresponding subspace, random exploration also proved enough for generating low-energy conformers close to the known endpoint of the conformational change of the LAO binding protein, lysozyme T4 and adenylate kinase.
\end{abstract}

\footnotetext{
*yves-henri.sanejouand@univ-nantes.fr
}

Keywords: Conformers, Normal modes, Distance constraints, Elastic Network Model, ROSETTA.

\section{Introduction}

It has been known for a while that domain motions involved in the function of a protein can be well described as a combination of motions along its lowest-frequency normal modes [1, 2, 3, 4, and this even when the normal modes are obtained using simplified protein models [1, 5, 6, 7, 8, 9, like the elastic network model (ENM) [10, 11, 12].

These results suggest that it should prove possible to predict the functional motion of a protein, since it amounts to guess the values of a few normal coordinates. Indeed, relevant conformers can be obtained by optimizing these values so as to match low-resolution X-ray crystallographic [13, 14, CryoEM [15, 16, 17] or SAXS [18, 19] data, to solve molecular replacement problems [17, 20] or to fulfill a set of experimentally determined distance constraints 21.

On the other hand, when additional experimental data are missing, normal mode coordinates can be used in order to explore the conformational landscape of a protein in the vicinity of a known structure, for instance through single mode-following techniques 22, 23, 24, 25. They can also be used so as to take advantage of the low-dimensionality of the subspace to be explored, in conjunction with various sampling methods [26], such as random exploration 27, energy minimization 228, 29, 30, 31, 
Monte Carlo Metropolis 32, 33, molecular dynamics 34, 35, 36, path planning protocols 37] or geometric simulations 38 .

In principle, they could also be used to perform straight jumps, that is, to go from a conformer to another one in a single step, noteworthy in the case of pairs of conformers with a $\mathrm{C}_{\alpha}$-root-mean-square difference (RMSd) of several Ångströms.

Indeed, it was shown in a previous study that, with the help of algorithms implemented in the ROSETTA software [39], it is possible to perform such jumps. Noteworthy, in the case of 14 large amplitude ( $\mathrm{RMSd}>2 \AA$ ) conformational changes, it proved possible to obtain conformers less than $1 \AA$ away from the target, that is, the known end-point of the conformational change [40]. Though encouraging, such successes proved rare, namely, $15 \%$ of the conformational changes considered, underlining the fact that using normal coordinates for generating accurate conformers far away from the initial one is not a straightforward task [41, 42, 43].

In the present study, a promising solution is proposed. Instead of building conformers using directly a set of normal coordinates, they are built in two steps, the conformer built with normal coordinates being just used for defining a large set of distance constraints, the final conformer being built so as to match them. Note that this approach amounts to transform the problem of generating accurate protein conformers using normal coordinates into a better known one: the distance-geometry problem [44, 45, 46].

\section{Methods}

\section{Building conformers using normal co- ordinates}

Conformers can be generated by displacing the $N$ atoms of a system along $n$ of its normal modes $(n \leq$ $3 N), \Delta r_{i}$, the displacement of coordinate $i$ being:

$$
\Delta r_{i}=\frac{1}{\sqrt{m_{i}}} \sum_{k=1}^{n} a_{i k} q_{k}
$$

where $q_{k}$ is the $\mathrm{k}^{t h}$ normal coordinate of the system, that is, the amplitude of the motion along mode $k$, $a_{i k}$ being the weight of mode $k$ for coordinate $i$, while $m_{i}$ is the mass of the corresponding atom.
In the present study, the elastic energy (eqn 4) of the conformers obtained using eqn 11 is usually minimized, under the following set of constraints:

$$
q_{k}=\text { cste }, \quad k=1 \ldots n
$$

In other words, during energy minimization, only the $3 N-n$ other coordinates are allowed to vary, as done in a number of previous studies [23, 24, 40.

In practice, conformer generation was performed with the dq_modes software, which is freely available on HAL (https://hal .archives-ouvertes. fr/hal-03144518), via the Software Heritage program [47.

The conformers thus obtained were not considered as is. Instead, they were used to specify NOElike restraints between all pairs of $\mathrm{C}_{\alpha}$ atoms of the protein considered, except for pairs of $\mathrm{C}_{\alpha}$ atoms belonging to consecutive residues along the sequence, the corresponding $\mathrm{C}_{\alpha}-\mathrm{C}_{\alpha}$ distances being not expected to vary significantly during a conformational change.

Conformers consistent with this set of distance restraints were then determined with the ROSETTA software 39], version 2018.12, using the RELAX protocol, a $1 \AA$ distance between the lower and upper NOE boundaries being assumed in order to take into account the lack of precision of the distances obtained above. Finally, all restraints were removed and an energy minimization was performed, with the parameters of the MINIMIZE protocol set to their default values.

Note that hereafter, for the sake of comparison between proteins of different sizes, minimum energies thus obtained are given per residue.

\section{Rebuilding protein conformational changes}

Like any motion, a conformational change of a protein, as for instance known by comparing structures $\mathrm{A}$ and B, can be described as a combination of motions along the normal modes of structure $\mathrm{A}$, that is, with values of the corresponding normal coordinates obtained as follows:

$$
q_{k}=\sum_{i=1}^{3 N} a_{i k} \sqrt{m_{i}} \Delta r_{i}
$$

$\Delta r_{i}$ being the difference between the values of the $\mathrm{i}^{t h}$ coordinate of the protein for structures $\mathrm{A}$ and 
B, structure B being first fitted onto structure A so as to have $q_{k}=0$ for the six zero-frequency modes associated to the overall translation and rotation of the protein. Note that, because the normal modes of a system form a basis set [48, 49], $I_{k}$, the percentage of involvement of mode $k$ in a given motion can be quantified as follows [50]:

$$
I_{k}=100 \frac{q_{k}^{2}}{\|\Delta \mathbf{r}\|}
$$

with $\sum^{3 N} I_{k}=100 \%$. Hereafter, it is assumed that mode $k$ is significantly involved in a conformational change if $I_{k}>5 \%$.

Reciprocally, using eqn 1 and the values of $n$ normal coordinates obtained through eqn 2, the conformational change of a protein can be partially rebuilt, the rebuilding being perfect when $n=3 N$.

Hereafter, like in our previous study 40, the rebuilding of protein conformational changes is performed using their $n$ lowest-frequency modes. Note however that conformers thus obtained are then optimized with ROSETTA, as described in the previous section, ten independant optimizations being performed for each conformer built using eqn 1 .

\section{Elastic network models}

Protein normal modes were calculated with a standard Elastic Network Model [10, 12, 51] (ENM), that is, a model where $V$, the (elastic) energy of the protein, is given by:

$$
V=\frac{1}{2} k_{e n m} \sum_{d_{i j}^{0}<R_{c}}\left(d_{i j}-d_{i j}^{0}\right)^{2}
$$

where $d_{i j}$ is the actual distance between atoms $i$ and $j, d_{i j}^{0}$ being their distance in the initial structure. $R_{c}$, a distance cutoff, is the only parameter of this ENM. Indeed, when, as assumed herein, $m_{i}=$ cste, $k_{e n m}$ is just an unit factor allowing for instance to specify the value of the lowest (nonzero) normal mode frequency of the system.

Hereafter, two flavors of this kind of ENM are considered, namely, the popular $\mathrm{C}_{\alpha}$-ENM [6, 12, 51, which is built using only $\mathrm{C}_{\alpha}$ atoms (with $\mathrm{R}_{c}=$ $12 \AA$ ), and the all-atom ENM noteworthy used by the Elnémo webserver [52] (with $\mathrm{R}_{c}=5 \AA$ ), where atoms belonging to a given residue are assumed to

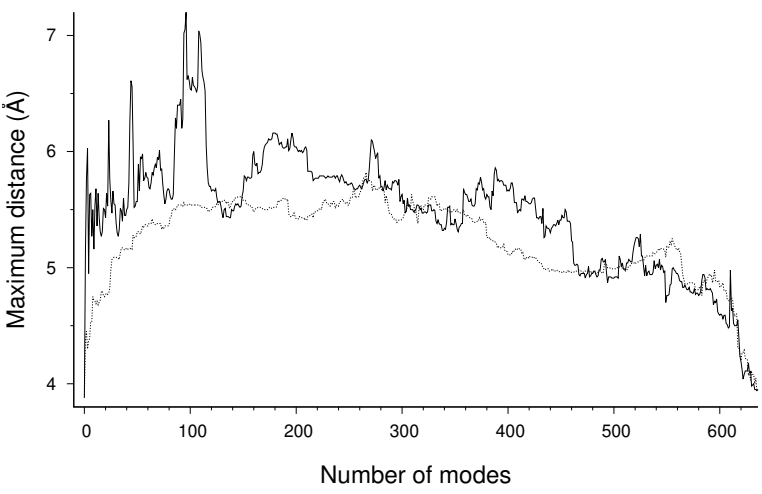

Figure 1: Maximum distance between consecutive $\mathrm{C}_{\alpha}$ atoms along the sequence, as a function of the number of $\mathrm{C}_{\alpha}$-ENM modes used to rebuild the conformational change of adenylate kinase. Plain line: straight rebuilding. Dotted line: when the elastic energy of the conformer is minimized, keeping the normal coordinates used for the rebuilding fixed.

behave like a rigid-body - the so-called RTB approximation [5, 53, 54, 55.

In practice, normal mode calculations were performed with the enm_modes software, which is freely available on HAL (https: //hal . archives-ouvertes.fr/hal-03142851), via the Software Heritage program [47].

\section{Results}

\section{The main bottleneck}

The main problem encountered when using only eqn 1 for generating protein conformers is illustrated in Figure 1. where the maximum distance between consecutive $\mathrm{C}_{\alpha}$ atoms along the sequence, observed during the rebuilding of the conformational change of adenylate kinase, as known by comparing the open (PDB 4AKE [56]) and closed (PDB 1AKE [57]) forms, is shown.

With a $\mathrm{C}_{\alpha}$-ENM of the open form of adenylate kinase, using eqn 1 and the value of the lowestfrequency normal coordinate (eqn 2 of the closed form, without any minimization of the elastic energy and no optimization by ROSETTA of the conformer thus obtained, the maximum distance between consecutive $\mathrm{C}_{\alpha}$ atoms is $5.2 \AA$, far from standard values, like the average one observed in the 
case of the open form, namely, $3.8 \pm 0.1 \AA$.

With the 102 lowest-frequency normal coordinates of the closed form, the maximum distance between consecutive $\mathrm{C}_{\alpha}$ atoms of the conformer becomes as high as $7.2 \AA$. However, its RMSd from the closed form is as low as $1.2 \AA$, in line with previous works showing that the conformational change of adenylate kinase can be well described as a combination of motions along its low-frequency modes 6. 5. So, while the overall conformational change of adenylate kinase is indeed very well described with the values of 102 normal coordinates, as illustrated in Figure 1. chemical details look then quite ugly. As a matter of fact, more than $\approx 450$ normal coordinates (70\% of them) are needed in order not to have any distance between consecutive $\mathrm{C}_{\alpha}$ atoms above $5 \AA$.

On the other hand, Figure 1 shows that minimizing the energy of the elastic energy of the conformer thus obtained, while keeping the considered normal coordinates fixed, as done in our previous study [40, does not improve significantly the chemical accuracy of the conformers, distances between consecutive $\mathrm{C}_{\alpha}$ atoms around $6 \AA$ being still observed.

In our previous study [40, in order to cope with this issue, all conformers were further optimized with ROSETTA 39, using harmonic restraints on the protein backbone. Hereafter, as mentioned in the Methods section, optimized conformers are obtained so as to match a large set of distance restraints. Interestingly, with such an approach, the chemical accuracy of the generated conformers is guaranteed, since it only depends upon the quality of the initial structure, that is, the structure used for the normal mode analysis.

While working with internal coordinates [58, 59, 60, 61 also secures the chemical accuracy of the generated conformers [32, 43], note that variations of a backbone internal coordinate can yield steric clashes far away along the sequence, a problem which is the counterpart of the problem with chemical accuracy occurring when working with cartesian coordinates.

\section{Choice of the kind of ENM}

As shown in Figure 2, in the case of the rebuilding of the conformational change of adenylate kinase, starting from the open form, using the values of

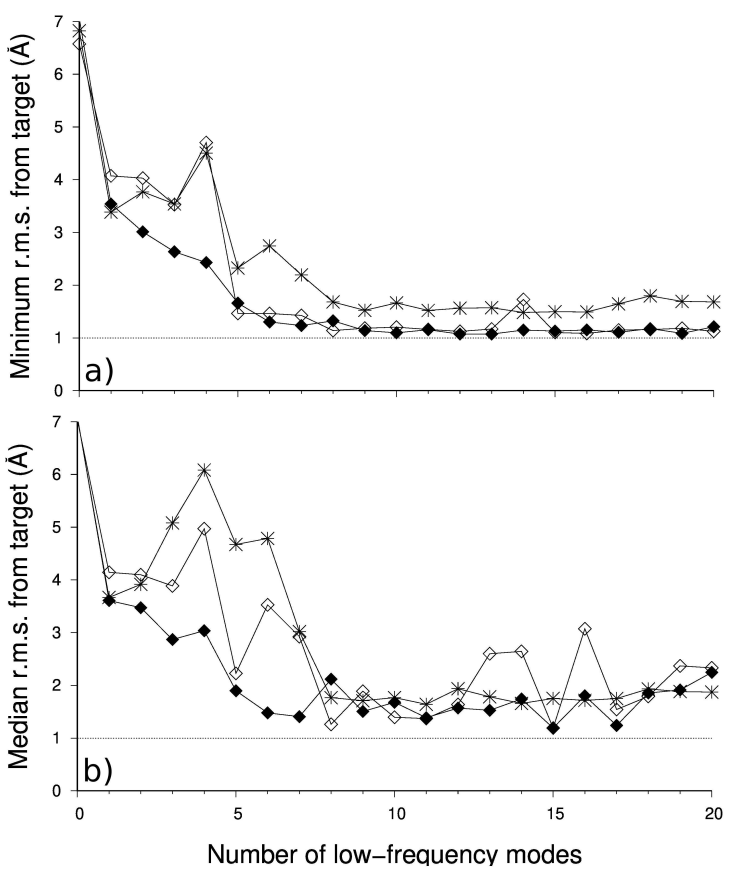

Figure 2: $\quad \mathrm{C}_{\alpha}$ root-mean-square deviation from adenylate kinase closed form (PDB 4AKE), as a function of the number of ENM modes used to rebuild the conformational change of adenylate kinase, starting from the open form (PDB 1AKE). a) Minimum and b) median values obtained after ten ROSETTA optimizations, using modes obtained with a $\mathrm{C}_{\alpha}$-ENM, with (open diamonds) or without (stars) minimization of the elastic energy, keeping the normal coordinates used for the rebuilding fixed; filled diamonds: using an all-atom ENM together with elastic energy minimization. Dotted line: corresponds to $1.0 \AA$.

less than 5-8 low-frequency normal coordinates of the closed form proves enough for obtaining optimized conformers less than $2 \AA$ away from the target. However, with the $\mathrm{C}_{\alpha}$-ENM and no preliminary minimization of the elastic energy of the conformers, even with 20 low-frequency normal coordinates, no optimized conformer less than $1.5 \AA$ away is obtained, while otherwise conformers $\approx 1.0$ $\AA$ away from the closed form are found (Fig. 2a).

Noteworthy, with the all-atom ENM, a median RMSd from the closed form of $1.5 \AA$ is observed with as little as six low-frequency normal coordinates (Fig. 2p). Moreover, with this model, the 


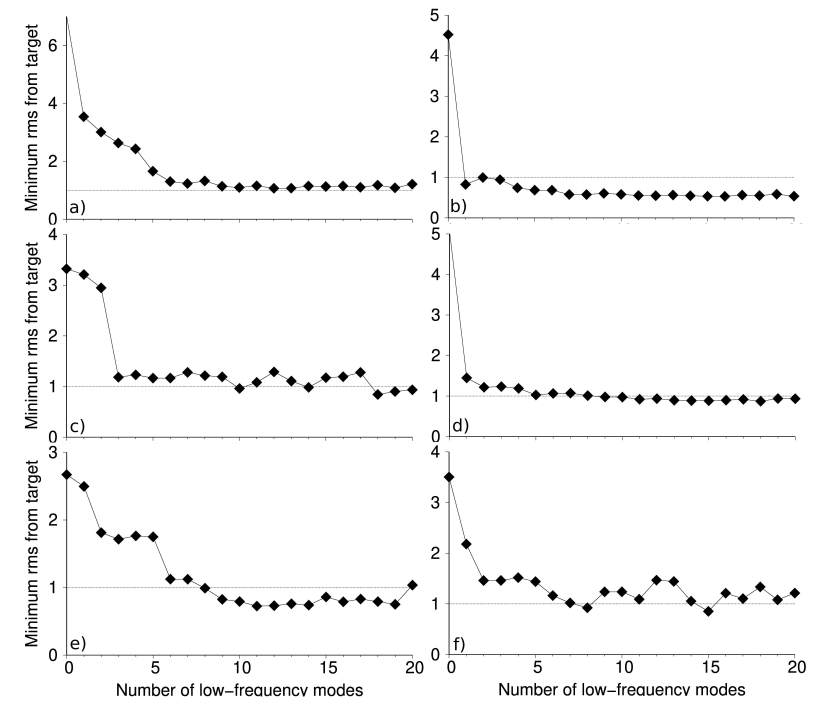

Figure 3: Minimum $\mathrm{C}_{\alpha}$ root-mean-square deviation from the target, as a function of the number of all-atom ENM modes used to rebuild the conformational change of: a) Adenylate kinase (4AKE $\rightarrow 1 \mathrm{AKE})$; b) LAO binding protein $(2 \mathrm{LAO} \rightarrow$ 1LST); c) Lysozyme T4 (177L $\rightarrow$ 178L); d) Glutamin binding protein $(1 \mathrm{GGG} \rightarrow 1 \mathrm{WDN})$; e) NF$\kappa \mathrm{B}(1 \mathrm{LEI} \rightarrow$ 1RAM); f) Maltose binding protein $(1 \mathrm{OMP} \rightarrow 1 \mathrm{ANF})$. Dotted line: corresponds to 1.0 $\AA$.

quality of the conformers obtained seems to depend less upon the exact number of low-frequency normal coordinates taken into account. This is the main reason why it was chosen for performing the rest of this work.

\section{Rebuilding conformational changes}

Figure 3 shows the results obtained when rebuilding the conformational change of six proteins, including classic cases like adenylate kinase [5, 6, 31, 62, lysozyme T4 63, 64, 65, the lysine/arginine/ornithine (LAO) 66, 60, 64] or the maltose 6, 10, 60] binding proteins.

The more spectacular result is obtained with the LAO binding protein (Fig. 3b). Indeed, providing the value of a single normal coordinate, namely, the lowest-frequency one, proves enough for building, starting from the open form (PDB 2LAO 66]), a conformer less than $1 \AA$ away from the closed form (PDB 1LST 66]). However, such a success is

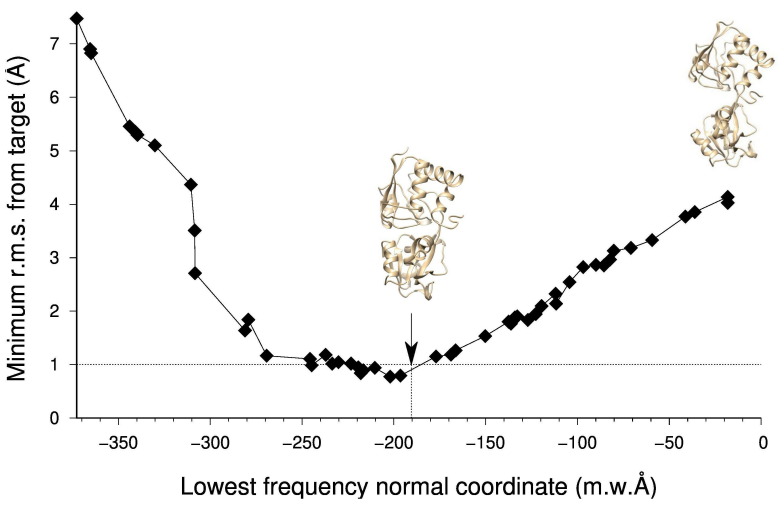

Figure 4: Minimum $\mathrm{C}_{\alpha}$ root-mean-square deviation from the LAO binding protein closed form (PDB 1LST), as a function of the value of the lowest-frequency normal coordinate used to build the conformers, starting from the open form (PDB 2LAO). The arrow pinpoints the value observed for the closed form. Dotted line: corresponds to $1.0 \AA$.

not surprising since the percentage of involvement (eqn 3) of the lowest-frequency normal mode of the open form in the conformational change of the LAO binding protein is very high $\left(I_{7}=86 \%\right)$.

A result almost as convincing is obtained in the case of the glutamin binding protein (Fig. 3d), since providing the value of the lowest-frequency normal coordinate allows to build a conformer $\approx$ $1.5 \AA$ away from the closed form (PDB 1WDN 67). Note that, in this case, the lowest-frequency mode is less involved in the conformational change $\left(I_{7}=76 \%\right)$, with another one being also significantly, though marginally, involved, namely, the second lowest-frequency one $\left(I_{8}=5 \%\right)$.

While three of them are needed in the case of Lysozyme T4 (Fig. 35), note that, in all six cases, conformers $\approx 1 \AA$ away from the target are obtained by specifying the values of no more than six low-frequency normal coordinates.

In this respect, NF- $\kappa \mathrm{B}$ is an interesting case. Indeed, providing less than six values does not allow to build conformers significantly less than $2 \AA$ away from the target (PDB 1RAM [68]). Adding the sixth value means that the highest-frequency mode found significantly involved in the conformational change is taken into account. Doing so, conformers $\approx 1 \AA$ away from the target can be built (Fig. 3e). So, though the involvement of mode 12 may seem 
Table 1: Average and lowest energy of the conformers found close to the target (in kcal/mole per residue). Conformers were randomly generated using between one and five low-frequency modes of the initial ones.

\begin{tabular}{|c|c|c|c|c|c|c|c|}
\hline Protein & $\langle\text { Energy }\rangle^{a}$ & Initial $\rightarrow$ Target & $\begin{array}{c}\text { RMSd } \\
(\AA)\end{array}$ & $\begin{array}{c}\text { Modes } \\
\text { used }^{b}\end{array}$ & $\begin{array}{c}\text { Number of } \\
\text { conformers }\end{array}$ & $\langle$ Energy $\rangle$ & $\begin{array}{c}\text { Lowest } \\
\text { energy }\end{array}$ \\
\hline LAO bp & $-3.27 \pm 0.03$ & 2LAO $\rightarrow$ 1LST & 3.4 & 7 & $38^{c}$ & $-3.2 \pm 0.1$ & -3.31 \\
Lysozyme T4 & $-2.78 \pm 0.05$ & $177 \mathrm{~L} \rightarrow 178 \mathrm{~L}$ & 4.7 & $7-9$ & $43^{d}$ & $-2.6 \pm 0.2$ & -2.84 \\
Adenylate kin. & $-3.02 \pm 0.03$ & $4 \mathrm{AKE} \rightarrow$ 1AKE & 7.1 & $7-11$ & $29^{e}$ & $-2.7 \pm 0.2$ & -2.94 \\
\hline
\end{tabular}

${ }^{a}$ Of the initial conformer, the average being over 100 relaxed conformations.

${ }^{b}$ Modes $1-6$ correspond to overall translation and rotation motions.

${ }^{c}$ With a RMSd from target $\leq 1.0 \AA$.

${ }^{d}$ With a RMSd from target $\leq 1.5 \AA$.

$e$ With a RMSd from target $\leq 2.0 \AA$.

low $\left(I_{12}=12 \%\right)$, the information brought by this mode proves important for the accurate rebuilding of the conformational change of $\mathrm{NF}-\kappa \mathrm{B}$.

\section{Single mode exploration}

If the value of a single normal coordinate is enough for rebuilding the conformational change of a protein, it is then straightforward, starting from the initial conformer, to build conformers close to the target when the structure of the latter is unknown, for instance by randomly picking values for the relevant normal coordinate.

This is illustrated in Figure 4, where 50 randomly chosen negative values of the lowest-frequency normal coordinate were considered, conformers being generated starting from the open form of the LAO binding protein. As above, for a given value of the normal coordinate, ten ROSETTA optimizations were performed. Among the 500 conformers thus obtained, $38(8 \%)$ were found less than $1 \AA$ away from the closed form. Moreover, compared to the energy of the open form, their average energy is similar, the lowest conformer energy observed being even lower than the average energy of the open form (see Table 1). Note that the values of the lowest-frequency normal coordinate used for generating such highly accurate conformers span a rather wide range, namely, between -245 and -196 massweighted $\AA$ (Fig. 4 ).

\section{Subspace exploration}

Random exploration of a subspace with less than ten dimensions can also prove efficient, as illustrated by the two following examples, where the sampling was however limited by choosing the right sign for the normal coordinates, that is, the sign observed for the target, as well as maximum values twice as large as the value known for the target.

In the case of Lysozyme T4, such random sampling of the values of the three lowest-frequency normal coordinates of a mutant with a wild-type conformation (PDB 177L 69) allowed to obtain 43 conformers (out of 1,000 trials) less than $1.5 \AA$ away from the target (PDB 178L 69]). Moreover, like in the case of the LAO binding protein, their average energy is rather low, the lowest conformer energy observed being also lower than the average energy of the initial one (see Table 1 ).

In the case of adenylate kinase, random sampling of the values of the five lowest-frequency normal coordinates of the open form allowed to obtain 22 conformers (out of 5,000) less than $2.0 \AA$ away from the closed form.

\section{Exploring with large jumps}

Using eqn 1, atomic displacements can be scaled, so as to generate conformers a given RMSd away from the initial one.

In the case of adenylate kinase, conformers 6-7 $\AA$ away from the open form (PDB 4AKE) were thus built, the scaling being performed after a random choice of the values of the five lowest-frequency normal coordinates. Note that, in this case, their sign was also chosen randomly.

First, 10,000 conformers were generated using eqn 1, and their elastic energy was minimized, keeping the five normal coordinates considered fixed. The 71 conformers found less than $3.5 \AA$ away 


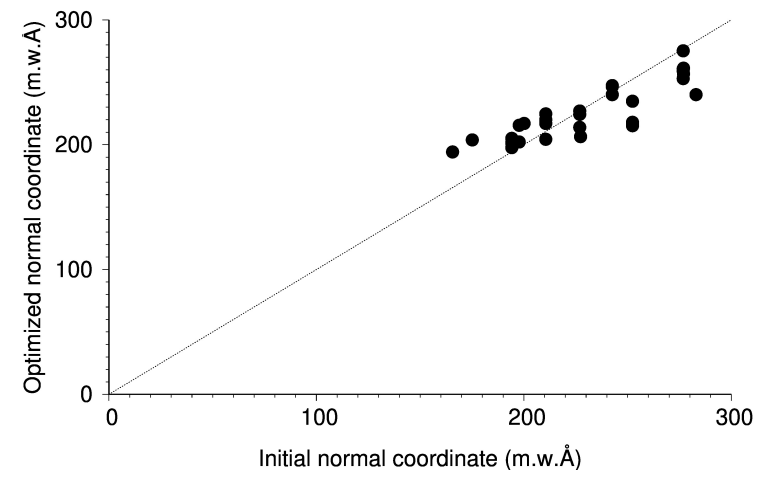

Figure 5: Optimized value of the lowest-frequency normal coordinate of adenylate kinase, as a function of its value before ROSETTA optimization, for conformers found less than $2.0 \AA$ away from the closed form, during a random exploration along the five lowest-frequency modes of the open form. $(0,0)$ corresponds to the open form. The dotted line, which indicates equal values, is a guide for the eye.

from the closed form were then optimized with ROSETTA, as done above, allowing to obtain 7 other conformers less than $2.0 \AA$ away from the target.

Though the average energy of the 29 conformers found close to the closed form of adenylate kinase is also low, at variance with both previous cases, the lowest conformer energy observed is significantly higher than the average energy of the open form (see Table 1). This is not due to the energy gap expected between open and closed forms since, according to ROSETTA, in spite of the fact that ligands are missing, the closed form has the lowest average energy, namely, $-3.17 \pm 0.04 \mathrm{kcal} / \mathrm{mole}$ per residue. It may mean instead that the sampling performed was not extensive enough (the generated conformer the closest to the closed form being still $1.6 \AA$ away), or that more than five normal coordinates should have been taken into account during the conformer generation process.

\section{The contribution of ROSETTA References}

As illustrated in Figure 5 for the case of adenylate kinase, probably as a consequence of the large number of restraints taken into account, the value of the lowest-frequency normal coordinate does not change much upon optimization by ROSETTA. This suggests that the approach introduced herein should work with various optimization methods, noteworthy those developed specifically for solving the distance geometry problem [44, 45, 46].

\section{Conclusion}

The approach proposed in the present study allowed to rebuild accurately (RMSd $\approx 1 \AA$ ) six large amplitude (RMSd > $3 \AA$ ) conformational changes, using the values of at most six low-frequency normal coordinates (Fig. 3).

This result noteworthy means that, in a predictive context, as a consequence of the lowdimensionality of the corresponding subspace, conformers close to the target can be obtained in a straightforward way, for instance by random sampling, as done herein for three classic cases, namely, the conformational changes of the LAO binding protein (Fig. 4), lysozyme T4 and adenylate kinase (Table 1).

More efficient algorithms should significantly speed up the conformer generation process. Note however that it is already a fairly quick one, generating an optimized conformer taking a few minutes only, on a single standard CPU.

So, a timely task left is to delineate the field of application of this approach. In the present study, a handful of proteins with clear-cut large amplitude domain motions were scrutinized. Proteins experiencing motions more arbitrary or of a more subtle nature need now to be considered.

\section{Acknowledgments}

This study is a sequel of the work undertaken with Dr Swapnil Mahajan during the BIP:BIP Projet d'Avenir (grant ANR-10-BINF-03-04).

[1] Harrison, R. (1984). Variational calculation of the normal modes of a large macromolecule: Methods and some initial results. Biopolymers 23, 2943-2949. 
[2] Brooks, B.R. \& Karplus, M. (1985). Normal modes for specific motions of macromolecules: application to the hinge-bending mode of lysozyme. Proc. Natl. Acad. Sci. USA 82, 4995-4999.

[3] Marques, O. \& Sanejouand, Y.H. (1995). Hinge-bending motion in citrate synthase arising from normal mode calculations. Proteins 23, 557-560.

[4] Perahia, D. \& Mouawad, L. (1995). Computation of low-frequency normal modes in macromolecules: improvements to the method of diagonalization in a mixed basis and application to hemoglobin. Comput. Chem. 19(3), 241246.

[5] Tama, F., Gadea, F.X., Marques, O. \& Sanejouand, Y.H. (2000). Building-block approach for determining low-frequency normal modes of macromolecules. Proteins 41(1), 1-7.

[6] Tama, F. \& Sanejouand, Y.H. (2001). Conformational change of proteins arising from normal mode calculations. Protein Eng., Des. Sel. 14(1), 1-6.

[7] Delarue, M. \& Sanejouand, Y.H. (2002). Simplified normal modes analysis of conformational transitions in DNA-dependant polymerases: the Elastic Network Model. J. Mol. Biol. 320, 1011-1024.

[8] Krebs, W.G., Alexandrov, V., Wilson, C.A., Echols, N., Yu, H. \& Gerstein, M. (2002). Normal mode analysis of macromolecular motions in a database framework: Developing mode concentration as a useful classifying statistic. Proteins 48(4), 682-695.

[9] Mahajan, S. \& Sanejouand, Y.H. (2015). On the relationship between low-frequency normal modes and the large-scale conformational changes of proteins. Arch. Biochem. Biophys. 567, 59-65.

[10] Tirion, M. (1996). Low-amplitude elastic motions in proteins from a single-parameter atomic analysis. Phys. Rev. Lett. 77, 19051908.
[11] Bahar, I., Atilgan, A.R. \& Erman, B. (1997). Direct evaluation of thermal fluctuations in proteins using a single-parameter harmonic potential. Fold. Des. 2, 173-181.

[12] Hinsen, K. (1998). Analysis of domain motions by approximate normal mode calculations. Proteins 33, 417-429.

[13] Tirion, M., ben Avraham, D., Lorenz, M. \& Holmes, K. (1995). Normal modes as refinement parameters for the F-actin model. Biophys. J. 68(1), 5-12.

[14] Chen, X., Wang, Q., Ni, F. \& Ma, J. (2010). Structure of the full-length Shaker potassium channel Kv1.2 by normal-mode-based X-ray crystallographic refinement. Proc. Natl. Acad. Sci. USA 107(25), 11352-11357.

[15] Tama, F., Valle, M., Frank, J. \& Brooks III, C.L. (2003). Dynamic reorganization of the functionally active ribosome explored by normal mode analysis and cryo-electron microscopy. Proc. Natl. Acad. Sci. USA 100(16), 9319-9323.

[16] Suhre, K., Navaza, J. \& Sanejouand, Y.H. (2006). Norma: a tool for flexible fitting of high resolution protein structures into low resolution electron microscopy derived density maps. Act. Cryst. D 62(9), 1098-1100.

[17] Delarue, M. \& Dumas, P. (2004). On the use of low-frequency normal modes to enforce collective movements in refining macromolecular structural models. Proc. Natl. Acad. Sci. USA 101(18), 6957-6962.

[18] Gorba, C. \& Tama, F. (2010). Normal mode flexible fitting of high-resolution structures of biological molecules toward SAXS data. Bioinformatics and biology insights 4, 43 .

[19] Panjkovich, A. \& Svergun, D.I. (2016). Deciphering conformational transitions of proteins by small angle X-ray scattering and normal mode analysis. Physical Chemistry Chem. Phys. 18(8), 5707-5719.

[20] Suhre, K. \& Sanejouand, Y.H. (2004). On the potential of normal mode analysis for solving difficult molecular replacement problems. Act. Cryst. D 60, 796-799. 
[21] Zheng, W. \& Brooks, B.R. (2005). Normal mode based prediction of protein conformational changes guided by distance constraints. Biophys. J. 88(5), 3109-3117.

[22] Wei, G., Mousseau, N. \& Derreumaux, P. (2002). Exploring the energy landscape of proteins: A characterization of the activationrelaxation technique. J. Chem. Phys. 117(24), 11379-11387.

[23] Miloshevsky, G.V. \& Jordan, P.C. (2006). The open state gating mechanism of gramicidin A requires relative opposed monomer rotation and simultaneous lateral displacement. Structure 14(8), 1241-1249.

[24] Floquet, N., Marechal, J.D., Badet-Denisot, M.A., Robert, C.H., Dauchez, M. \& Perahia, D. (2006). Normal mode analysis as a prerequisite for drug design: application to matrix metalloproteinases inhibitors. FEBS Lett. $\mathbf{5 8 0}(22), 5130-5136$.

[25] Batista, P.R., Pandey, G., Pascutti, P.G., Bisch, P.M., Perahia, D. \& Robert, C.H. (2011). Free energy profiles along consensus normal modes provide insight into HIV-1 protease flap opening. J. Chem. Theory Comput. 7(8), 2348-2352.

[26] Krieger, J.M., Doruker, P., Scott, A.L., Perahia, D. \& Bahar, I. (2020). Towards gaining sight of multiscale events: utilizing network models and normal modes in hybrid methods. Curr. Opin. Struct. Biol. 64, 34-41.

[27] Venkatraman, V. \& Ritchie, D.W. (2012). Flexible protein docking refinement using pose-dependent normal mode analysis. Proteins: Struct., Funct., Bioinf. 80(9), 22622274 .

[28] Cavasotto, C.N., Kovacs, J.A. \& Abagyan, R.A. (2005). Representing receptor flexibility in ligand docking through relevant normal modes. J. Am. Chem. Soc. 127(26), 96329640 .

[29] May, A. \& Zacharias, M. (2008). Energy minimization in low-frequency normal modes to efficiently allow for global flexibility during systematic protein-protein docking. Proteins: Struct., Funct., Bioinf. 70(3), 794-809.

[30] Moal, I.H. \& Bates, P.A. (2010). Swarmdock and the use of normal modes in protein-protein docking. International journal of molecular sciences 11, 3623-3648.

[31] Kurkcuoglu, Z., Bahar, I. \& Doruker, P. (2016). ClustENM: ENM-based sampling of essential conformational space at full atomic resolution. J. Chem. Theory Comput. .

[32] Noguti, T. \& Gō, N. (1985). Efficient monte carlo method for simulation of fluctuating conformations of native proteins. Biopolymers: Original Research on Biomolecules 24(3), 527-546.

[33] Miloshevsky, G.V. \& Jordan, P.C. (2007). Open-state conformation of the KcsA $\mathrm{K}^{+}$ channel: Monte Carlo normal mode following simulations. Structure 15(12), 1654-1662.

[34] Durup, J. (1991). Protein molecular dynamics constrained to slow modes: theoretical approach based on a hierarchy of local modes with a set of holonomic constraints; the method and its tests on citrate synthase. $J$. Phys. Chem. 95(4), 1817-1829.

[35] Elezgaray, J. \& Sanejouand, Y.H. (1998). Modeling large-scale dynamics of proteins. Biopolymers 46, 493-501.

[36] Costa, M.G., Batista, P.R., Bisch, P.M. \& Perahia, D. (2015). Exploring free energy landscapes of large conformational changes: molecular dynamics with excited normal modes. $J$. Chem. Theory Comput. 11(6), 2755-2767.

[37] Kirillova, S., Cortes, J., Stefaniu, A. \& Simeon, T. (2008). An NMA-guided path planning approach for computing largeamplitude conformational changes in proteins. Proteins 70(1), 131-143.

[38] Jimenez-Roldan, J., Freedman, R., Römer, R. \& Wells, S.A. (2012). Rapid simulation of protein motion: merging flexibility, rigidity and normal mode analyses. Phys. Biol. 9(1), 016008 . 
[39] O’Meara, M.J., Leaver-Fay, A., Tyka, M.D. Stein, A., Houlihan, K., DiMaio, F., Bradley, P., Kortemme, T., Baker, D., Snoeyink, J. et al. (2015). Combined covalent-electrostatic model of hydrogen bonding improves structure prediction with Rosetta. J. Chem. Theory Comput. 11(2), 609-622.

[40] Mahajan, S. \& Sanejouand, Y.H. (2017). Jumping between protein conformers using normal modes. J. Comput. Chem. 38, 16221630 .

[41] Yang, Q. \& Sharp, K.A. (2009). Building alternate protein structures using the elastic network model. Proteins: Struct., Funct., Bioinf. 74(3), 682-700.

[42] Ahmed, A., Rippmann, F., Barnickel, G. \& Gohlke, H. (2011). A normal mode-based geometric simulation approach for exploring biologically relevant conformational transitions in proteins. J. Chem. Inf. Model. 51(7), 16041622 .

[43] Frezza, E. \& Lavery, R. (2019). Internal coordinate normal mode analysis: A strategy to predict protein conformational transitions. $J$. Phys. Chem. B 123(6), 1294-1301.

[44] Wagner, G., Braun, W., Havel, T.F., Schaumann, T., Gō, N. \& Wüthrich, K. (1987). Protein structures in solution by nuclear magnetic resonance and distance geometry: the polypeptide fold of the basic pancreatic trypsin inhibitor determined using two different algorithms, DISGEO and DISMAN. $J$. Mol. Biol. 196(3), 611-639.

[45] Moré, J.J. \& Wu, Z. (1999). Distance geometry optimization for protein structures. Journal of Global Optimization 15(3), 219-234.

[46] Malliavin, T.E., Mucherino, A., Lavor, C. \& Liberti, L. (2019). Systematic exploration of protein conformational space using a distance geometry approach. J. Chem. Inf. Model. 59(10), 4486-4503.

[47] Di Cosmo, R. \& Zacchiroli, S. (2017). Software heritage: Why and how to preserve software source code. In iPRES 2017-14th International Conference on Digital Preservation, pages $1-10$.
[48] Wilson, E., Decius, J. \& Cross, P. (1955). Molecular Vibrations. McGraw-Hill, New York.

[49] Goldstein, H. (1956). Classical Mechanics. Addison-Wesley, Reading, MA.

[50] Ma, J. \& Karplus, M. (1997). Ligand-induced conformational changes in ras p21: a normal mode and energy minimization analysis. $J$. Mol. Biol. 274, 114-131.

[51] Doruker, P., Atilgan, A.R. \& Bahar, I. (2000). Dynamics of proteins predicted by molecular dynamics simulations and analytical approaches: Application to $\alpha$-amylase inhibitor. Proteins: Struct., Funct., Bioinf. 40(3), 512524.

[52] Suhre, K. \& Sanejouand, Y.H. (2004). Elnémo: a normal mode server for protein movement analysis and the generation of templates for molecular replacement. Nucl. Ac. Res. 32, W610-W614.

[53] Durand, P., Trinquier, G. \& Sanejouand, Y.H. (1994). A new approach for determining lowfrequency normal modes in macromolecules. Biopolymers 34, 759-771.

[54] Li, G. \& Cui, Q. (2002). A coarse-grained normal mode approach for macromolecules: an efficient implementation and application to $\mathrm{Ca}^{2+}$-ATPase. Biophys. J. 83(5), 2457-2474.

[55] Hoffmann, A. \& Grudinin, S. (2017). Nolb: Nonlinear rigid block normal-mode analysis method. J. Chem. Theory Comput. 13(5), 2123-2134.

[56] Müller, C., Schlauderer, G., Reinstein, J. \& Schulz, G.E. (1996). Adenylate kinase motions during catalysis: an energetic counterweight balancing substrate binding. Structure 4(2), $147-156$.

[57] Müller, C.W. \& Schulz, G.E. (1992). Structure of the complex between adenylate kinase from Escherichia coli and the inhibitor Ap5A refined at $1.9 \AA$ resolution: A model for a catalytic transition state. J. Mol. Biol. 224(1), 159177. 
[58] Noguti, T. \& Go, N. (1982). Collective variable description of small-amplitude conformational fluctuations in a globular protein. Nature 296, 776-778.

[59] Levitt, M., Sander, C. \& Stern, P. (1983). Normal-mode dynamics of a protein: Bovine pancreatic trypsin inhibitor. Int. J. Quant. Chem. 10, 181-199.

[60] López-Blanco, J.R., Aliaga, J.I., QuintanaOrtí, E.S. \& Chacón, P. (2014). iMODS: internal coordinates normal mode analysis server. Nucleic Acids Res. 42(W1), W271-W276.

[61] Tirion, M.M. \& ben Avraham, D. (2015). Atomic torsional modal analysis for highresolution proteins. Phys. Rev. E 91(3), 032712 .

[62] Maragakis, P. \& Karplus, M. (2005). Large amplitude conformational change in proteins explored with a plastic network model: adenylate kinase. J. Mol. Biol. 352(4), 807-822.

[63] De Groot, B., Hayward, S., Van Aalten, D. Amadei, A. \& Berendsen, H. (1998). Domain motions in bacteriophage T4 lysozyme: a comparison between molecular dynamics and crystallographic data. Proteins: Struct., Funct., Bioinf. 31(2), 116-127.

[64] Nicolay, S. \& Sanejouand, Y.H. (2006). Functional modes of proteins are among the most robust. Phys. Rev. Lett. 96(7), 078104.

[65] Zacharias, M. (2008). Combining elastic network analysis and molecular dynamics simulations by hamiltonian replica exchange. $J$. Chem. Theory Comput. 4(3), 477-487.

[66] Oh, B., Pandit, J., Kang, C., Nikaido, K., Gokcen, S., Ames, G. \& Kim, S. (1993). Three-dimensional structures of the periplasmic lysine/arginine/ornithine-binding protein with and without a ligand. J. Biol. Chem. 268, 11348-11355.

[67] Sun, Y.J., Rose, J., Wang, B.C. \& Hsiao, C.D. (1998). The structure of glutamine-binding protein complexed with glutamine at $1.94 \AA$ resolution: comparisons with other amino acid binding proteins. J. Mol. Biol. 278(1), 219229.
[68] Chen, Y.Q., Ghosh, S. \& Ghosh, G. (1998). A novel DNA recognition mode by the NF$\kappa \mathrm{B}$ p65 homodimer. Nature structural biology 5(1), 67-73.

[69] Faber, H.R. \& Matthews, B.W. (1990). A mutant T4 lysozyme displays five different crystal conformations. Nature 348, 263-266. 